\title{
Working memory for language is not special: Evidence for an articulatory loop for novel stimuli
}

\author{
Margaret Wilson And GLenn Fox \\ University of California, Santa Cruz, California
}

\begin{abstract}
The "articulatory loop" for rehearsal of verbal materials in working memory has been shown not to be a unique hard-wired structure associated with spoken language. Specifically, a parallel rehearsal process for sign language occurs in fluent signers. Here we show that the same rehearsal process can occur for unfamiliar, nonmeaningful body movements. We conclude that working memory maintenance does not rely on a dedicated architecture, but instead involves the strategic recruitment of resources as needed for the task demands.
\end{abstract}

Rehearsal of verbal materials in working memory has long seemed to have a privileged status in comparison with other aspects of working memory. It is fast, efficient, more or less universal, and has yielded to scientific analysis in a way that other forms of working memory have not. Specifically, the Baddeley model of the articulatory loop (Baddeley, 1986, 1995; Baddeley \& Hitch, 1974, 1994), though often challenged on its details, still appears to capture the essentials of how verbal rehearsal works (Wilson, 2001a). Briefly described, the articulatory loop involves covert motoric repetition as a way of repeatedly refreshing a storage buffer. In contrast, working memory maintenance for other types of materials (e.g., nonnamable shapes) does not seem to be subserved by a similar mechanism (Wilson, 2001a).

This might be taken to suggest that the human evolutionary history with spoken language has hard-wired into the brain a dedicated mechanism, which uses speechbased representations for working memory. However, research with American Sign Language (ASL) has shown this to not be the case. The signature effects of the articulatory loop, including the phonological similarity effect, the word length effect, and the articulatory suppression effect, have all been shown to have exact parallels in ASL (Krakow \& Hanson, 1985; Poizner, Bellugi, \& Tweney, 1981; Wilson \& Emmorey, 1997a, 1997b, 1998, 2003). This indicates, at a minimum, that speech is not privileged. Instead, an "articulatory loop" can develop on the basis of the sensorimotor properties of radically different forms of language.

The next question, then, is: What are the necessary conditions for the development of an articulatory loop? Is the articulatory loop necessarily tied to language? Does it require lifelong practice with a complex input-output system? Or can any motorically enacted stimulus lend itself to the use of an articulatory loop? If the latter is true, it would suggest that the articulatory loop observed for language is not a "mechanism" at all (either genetically hard-wired or ontogenetically developed), but is, rather, the strategic recruitment of sensory and motor resources.

Here we report four experiments showing that working memory for novel gestures yields three signature effects of the articulatory loop.

\section{The Problem of Verbal Labeling}

Previous attempts to study working memory for nonmeaningful body movements have been hindered by the strong tendency of subjects to invent verbal labels. When this happens, subjects are no longer, strictly speaking, remembering body movements; they are remembering verbal labels from which they can reconstruct the body movements. In order to study working memory for gestures, it is necessary to minimize this tendency to label. Articulatory suppression is a poor option, because it introduces too many simultaneous activities when combined with the selective interference manipulation described below, and also because it does not fully eliminate phonological recoding (Baddeley, 1986).

In order to minimize verbal labeling, we developed a new method that relies on using unique, or nearly unique, items on each trial. Traditionally, immediate serial recall tasks use sampling-with-replacement from a small set of items to prime all items equally in long-term memory, thus forcing the subject to rely on an immediate memory trace of the particular trial. However, this is unnecessary for unfamiliar items with no preexisting long-term-memory representation; furthermore, it is counterproductive, for it is precisely this repetition that allows subjects to develop a verbal labeling strategy.

In the present experiments, instead, a large number of stimuli are used, with minimal reuse. This is done by generating all possible permutations of gestures from a set of locations, movements, and handshapes. (This approach resembles the composition of signs in natural signed languages, and is far easier than attempting to generate a large

M.Wilson, mlwilson@ucsc.edu 
number of holistically unique gestures.) Specifically, in these experiments, stimuli were composed from three handshapes, three locations, and three movements, resulting in 27 unique stimuli (see Figure 1). Note that this does not entirely prevent verbal labeling, and in fact subjects almost certainly use some verbal labeling, even with these stimuli. (Observation of subjects' mouth movements during the task, as well as subjects' spontaneous self-reports, suggests that subjects often choose to verbally encode the movement parameter.) However, in a trial with even as few as three stimuli, it is essentially impossible for subjects to verbally encode all parameters of all three gestures (a total of 9 elements, presented within $3 \mathrm{sec}$ ). Likewise, it is essentially impossible for them to invent a unique label for each whole gesture (e.g., a circular movement, with the " 5 " handshape on the forehead), since these are many in number, and they change from trial to trial. Thus, the intention behind this method is to force subjects to rely at least in part on covertly recreating the gestures at a motoric level.

\section{GENERAL METHOD}

Students from UCSC psychology classes received course credit for participation. None knew ASL or any other sign language.

Three handshapes, three locations, and three movements were combined parametrically to create 27 gestures (see Figure 1), each of which was used, at most, 6 times in a single experiment. Each stimulus was videotaped separately. Stimuli were combined into "lists" of 3 gestures so that each handshape, location, and movement occurred only once in a list.

Each trial involved the presentation of a list by showing the three video clips at a rate of one per second on a computer screen. Nine practice trials were always given before the main experiment began.

In Experiments 1-3, after the third clip, the subject reproduced the three gestures as closely as possible, with responses videotaped for later scoring. One point was assigned for each element of each gesture recalled in the correct serial position (for a possible total of 9 points per trial). Experiment 4, in contrast, used a probe paradigm, in which subjects were cued to report a particular item from the list. This was done by presenting a probe item taken from the list $.67 \mathrm{sec}$ after the end of the list. The subject's task was to reproduce the gesture that had occurred after the probe gesture in the list (for a possible total of 3 points per trial).

\section{EXPERIMENT 1 Similarity Effect}

One of the key effects in the verbal working-memory literature is the phonological similarity effect, in which lists of words that sound similar are more difficult to recall than are lists of diverse words (Baddeley, 1986). With ASL, a parallel effect has been found, in which lists of signs with similar handshapes are more difficult to recall than are lists with diverse handshapes (Krakow \& Hanson, 1985; Poizner et al., 1981; Wilson \& Emmorey, 1997a). In this experiment, we tested for a handshape similarity effect in a subject sample of 20 nonsigners.

A total of 27 lists using the three visually dissimilar handshapes shown in Figure 1 were compared with 27 lists using three visually similar handshapes (five fingers extended, four fingers extended, thumb and first two fingers extended). Similar and dissimilar lists were alternated.
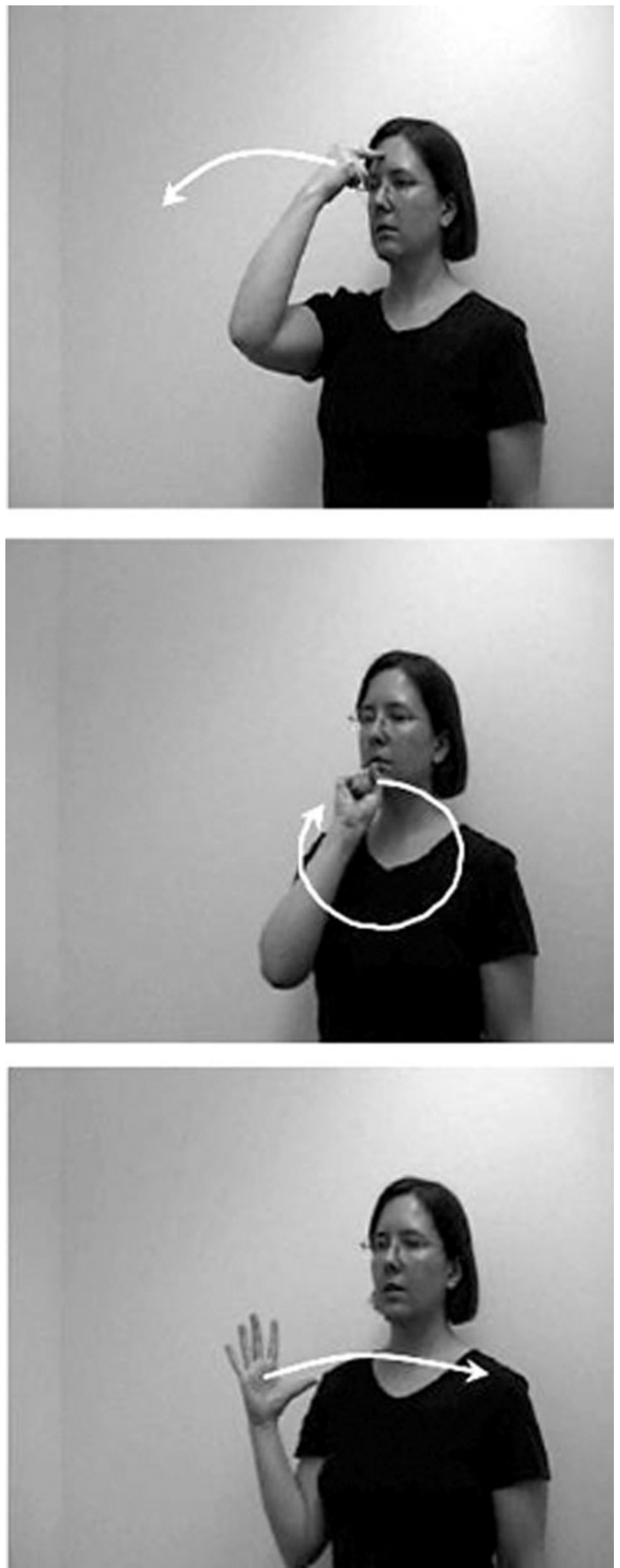

Figure 1. Three examples of gestures used in the experiments. Arrows indicate direction of movement shown on video. Gestures comprised all possible permutations of three locations (forehead, chin, or chest), three movements (out, circle, or across), and three handshapes (index finger, fist, or five fingers). 
Performance was better for the dissimilar lists $(M=$ $86.2 \%, S E=1.6)$ than for the similar lists $(M=82.8 \%$, $S E=1.8)$, a statistically significant difference $[t(19)=$ $3.00, p=.007]$.

\section{EXPERIMENT 2 Suppression Effect}

A second important effect in the working memory literature is the articulatory suppression effect, in which a simple, repetitive movement with relevant articulators disrupts rehearsal in working memory. Thus, repetitive mouth movement disrupts memory for spoken language materials (Baddeley, 1986), and repetitive hand movement disrupts memory for sign language materials (Wilson \& Emmorey, 1998). Here we tested for a manual suppression effect in a subject sample of 12 nonsigners.

A total of 54 lists were shown, all using the stimuli shown in Figure 1. On half the trials, subjects were asked to perform a meaningless hand movement continuously throughout the stimulus presentation. This consisted of the thumb touching the middle finger of each hand, and the dominant hand repeatedly circling and then tapping the nondominant hand. (This is the movement used by Wilson \& Emmorey, 1998, for manual suppression with signers.) Suppression trials and no-suppression trials were alternated for a total of 27 trials in each condition.

Performance was better with no suppression $(M=$ $87.1 \%, S E=2.6)$ than with suppression $(M=80.8 \%$, $S E=3.6)$, a statistically significant difference $[t(11)=$ $3.57, p=.004]$.

\section{EXPERIMENT 3 Length Effect}

A third signature effect is the word length effect, which perhaps should be more broadly called the stimulus length effect. Lists of long words yield worse performance than lists of short words (Baddeley, 1986), and lists of long signs yield worse performance than lists of short signs (Wilson \& Emmorey, 1998).

However, some care is needed in constructing long versus short gestures. In the sign language experiments of Wilson and Emmorey (1998), signs that covered long distances were compared with signs that had only short local movement. One difficulty with this for unfamiliar stimuli is that long-movement gestures may be perceived as more dramatic and visually distinctive than short-movement gestures. An alternative possibility is to add extra movement trajectories to make some stimuli longer, without resulting in a larger gesture. The problem here, though, is that this would be adding further elements to be remembered, which could spuriously cause the long items to be more difficult to remember.

To avoid both these problems, we created long gestures by simply doubling the movements used for the short gestures. Any given trial consisted entirely of double or of single movements, so that there was no additional informational burden in either condition. Instead, the only difference between the two conditions is that the double movements take longer to produce, and therefore, presumably, longer to mentally rehearse. Using these stimuli, we tested for a length effect of gestures in a subject sample of 8 nonsigners.

A total of 27 lists using the stimuli shown in Figure 1 were compared with 27 lists in which each movement was performed twice in rapid succession. Lists with short (single-movement) and long (double-movement) stimuli were alternated.

Performance was better for the short-stimuli lists $(M=$ $89.5 \%, S E=1.9)$ than for the long-stimuli lists $(M=$ $83.3 \%, S E=2.4)$, a statistically significant difference $[t(7)=4.47, p=.003]$.

\section{EXPERIMENT 4 Length Effect With Probe Paradigm}

The length effect found in Experiment 3 could be a result of the length of time required for the subject to reproduce the stimuli, during which additional forgetting could occur. This has been shown to contribute to the length effect observed for spoken language (Avons, Wright, \& Pammer, 1994). To control for this, we next tested for a length effect in a paradigm in which subjects are required to recall only one stimulus per trial, by presenting a probe item and requiring recall of the subsequent item in the list (Wilson \& Emmorey, 1998). Because subjects do not know ahead of time which stimulus will be probed, and because serial order information is still required, this method probes memory for the whole sequence. Using this method, we tested for a length effect of gestures in a subject sample of 16 nonsigners. The method followed that of Experiment 3, with the exception of the use of the probe paradigm described in the General Method.

Performance was better for the short-stimuli lists $(M=87.6 \%, S E=2.0)$ than for the long-stimuli lists $(M=85.1 \%, S E=2.4)$, a statistically significant difference $[t(15)=2.13, p=.05]$.

\section{DISCUSSION}

Four experiments show that, when care is taken to establish conditions that discourage alternate strategies (notably, verbal strategies), immediate serial recall for gestures shows effects parallel to those of immediate serial recall for words and for signs. Specifically, it shows evidence of sensorimotor-based rehearsal equivalent to the articulatory loop for language rehearsal. To the extent that this pattern of data in the literatures on speech and sign is taken to support a two-part loop, including a quasisensory storage buffer and a motoric rehearsal process (Baddeley, 1986; Wilson, 2001a), the same conclusion appears to apply equally to the stimuli tested here.

This is notable, first because it involves nonlinguistic stimuli and second because it involves stimuli not commonly seen or produced in daily life and, therefore, essentially new to these subjects. This indicates that the equivalent of an articulatory loop can be created on the fly, in response to appropriate task demands.

The rather small size of the effects found here is likely due to these subjects' lack of fluency in rehearsing these 
particular stimuli. As has been repeatedly shown, for rapid, automatic rehearsal in working memory (Thorn \& Gathercole, 2001), stimulus familiarity matters. Furthermore, as noted earlier, subjects employ verbal labeling to some degree; this can be expected to weaken effects through the use of multiple strategies. What is remarkable is that subjects show any evidence at all of manual motoric rehearsal for these unfamiliar stimuli and, furthermore, that this rehearsal appears to reflect the same articulatory loop structure as is found for the highly practiced domain of language. This suggests that with practice, robust articulatory loops could develop for any number of action domains (cf. Reisberg, Rappaport, \& O’Shaughnessy, 1984).

These findings further suggest that a key element that separates active rehearsal in working memory from other forms of maintenance, such as for abstract visual patterns, is whether the stimuli lend themselves to imitation - that is, to motor encoding that in some way recreates or mirrors the stimulus itself. Independent evidence shows that stimuli that can be imitated are processed differently than are other sorts of stimuli, and that they involve tight reciprocal connections between input representations and output representations (Loula, Prasad, Harber, \& Shiffrar, 2005; Prinz, 1997; Wilson, 2001b, 2006; Wilson \& Knoblich, 2005). This way of conceptualizing the issue predicts that any stimulus that can be encoded with equivalent sensory and motor representations will support an articulatory loop, but that stimuli that cannot be so encoded will not show evidence of this strategy. The absence of any coherent body of evidence for an articulatory loop for nonnameable shapes, after decades of research, suggests that this prediction will hold (Wilson, 2001a).

The findings reported here also help to clarify how working memory subdivides into separate domains. Classically, the major division within working memory is between the verbal and visuospatial domains (Baddeley, 1986). However, this formulation contains an ambiguity (Wilson \& Emmorey, 1997a): Is the distinction based on modality (i.e., auditory vs. visual), or on stimulus structure (i.e., motoric vs. not motoric)? The present findings, as well as previous findings on sign language, show that visuospatial working memory can in fact behave like verbal working memory, at least when the stimuli can be motorically reproduced. This suggests that there are potentially at least four domains to be considered: motoric auditory (e.g., speech); motoric visual (e.g., signs and gestures); nonmotoric auditory (e.g., environmental sounds); and nonmotoric visual (e.g., nonnamable shapes). Research on irrelevant sensory input with speech and sign language shows that perceptual resources are engaged even for motorically based working memory (Colle \& Welsh, 1976; Wilson \& Emmorey, 2003). Thus, it seems likely that working memory for signs and gestures shares resources with more general visual working memory, and working memory for speech shares resources with more general auditory working memory.

Finally, we conclude with the point that these data suggest a view of working memory as a flexible entity. Rather than involving hard-wired and dedicated components, working memory may instead consist of the strategic re- cruitment of cognitive resources, determined on the fly by the immediate demands of the task.

\section{AUTHOR NOTE}

We thank Jack Day, Alicia Helion, Greg Reaume, and Alexis Spenser for their assistance. Correspondence concerning this article should be addressed to M. Wilson, Department of Psychology, University of California, Santa Cruz, CA 95064 (e-mail: mlwilson@ucsc.edu).

\section{REFERENCES}

Avons, S. E., Wright, K. L., \& Pammer, K. (1994). The word-length effect in probed and serial recall. Quarterly Journal of Experimental Psychology, 47A, 207-231.

Baddeley, A. D. (1986). Working memory. New York: Oxford University Press.

BAdDELEY, A. D. (1995). Working memory. In M. S. Gazzaniga (Ed.), The cognitive neurosciences (pp. 755-764). Cambridge, MA: MIT Press.

BADDEley, A. D., \& Hitch, G. (1974). Working memory. In G. H. Bower (Ed.), The psychology of learning and motivation (Vol. 8, pp. 647-667). Hillsdale, NJ: Erlbaum.

BADDEley, A. D., \& Hitch, G. (1994). Developments in the concept of working memory. Neuropsychology, 8, 485-493.

Colle, H. A., \& Welsh, A. (1976). Acoustic masking in primary memory. Journal of Verbal Learning \& Verbal Behavior, 15, 17-31.

KRAKOW, R. A., \& Hanson, V. L. (1985). Deaf signers and serial recall in the visual modality: Memory for signs, fingerspelling, and print. Memory \& Cognition, 13, 265-272.

Loula, F., Prasad, S., Harber, K., \& Shiffrar, M. (2005). Recognizing people from their movement. Journal of Experimental Psychology: Human Perception \& Performance, 31, 210-220.

Poizner, H., Bellugi, U., \& Tweney, R. (1981). Processing of formational, semantic, and iconic information in American Sign Language. Journal of Experimental Psychology: Human Perception \& Performance, 7, 1146-1159.

PRINZ, W. (1997). Perception and action planning. European Journal of Cognitive Psychology, 9, 129-154.

Reisberg, D., Rappaport, I., \& O'Shaughnessy, M. (1984). Limits of working memory: The digit digit-span. Journal of Experimental Psychology: Learning, Memory, \& Cognition, 10, 203-221.

Thorn, A. S. C., \& Gathercole, S. E. (2001). Language differences in verbal short-term memory do not exclusively originate in the process of subvocal rehearsal. Psychonomic Bulletin \& Review, 8, 357-364.

WILSON, M. (2001a). The case for sensorimotor coding in working memory. Psychonomic Bulletin \& Review, 8, 44-57.

WiLson, M. (2001b). Perceiving imitatable stimuli: Consequences of isomorphism between input and output. Psychological Bulletin, 127, 543-553.

WiLson, M. (2006). Covert imitation: How the body schema acts as a prediction device. In G. Knoblich, I. M. Thornton, M. Grosjean, \& M. Shiffrar (Eds.), Human body perception from the inside out (pp. 211-228). Oxford: Oxford University Press.

Wilson, M., \& Emmorey, K. (1997a). A visuospatial "phonological loop" in working memory: Evidence from American Sign Language. Memory \& Cognition, 25, 313-320.

WiLSON, M., \& EMMOREY, K. (1997b). Working memory for sign language: A window into the architecture of the working memory system. Journal of Deaf Studies \& Deaf Education: Special Issue on Memory \& Cognition, 2, 121-130.

Wilson, M., \& EMmorey, K. (1998). A "word length effect" for sign language: Further evidence on the role of language in structuring working memory. Memory \& Cognition, 26, 584-590.

Wilson, M., \& EMmorey, K. (2003). The effect of irrelevant visual input on working memory for sign language. Journal of Deaf Studies \& Deaf Education, 8, 97-103.

Wilson, M., \& KNOBLICH, G. (2005). The case for motor involvement in perceiving conspecifics. Psychological Bulletin, 131, 460-473.

(Manuscript received January 30, 2006; revision accepted for publication July 17, 2006.) 